\title{
Dendrimers as tunable vectors of drug delivery systems and biomedical and ocular applications
}

This article was published in the following Dove Press journal:

International Journal of Nanomedicine

22 December 2015

Number of times this article has been viewed

\author{
Marina Kalomiraki' \\ Kyriaki Thermos ${ }^{2}$ \\ Nikos A Chaniotakis' \\ 'Laboratory of Analytical Chemistry, \\ Department of Chemistry, \\ ${ }^{2}$ Department of Pharmacology, \\ School of Medicine, University of \\ Crete Voutes, Heraklion, Greece
}

\begin{abstract}
Dendrimers are large polymeric structures with nanosize dimensions (1-10 nm) and unique physicochemical properties. The major advantage of dendrimers compared with linear polymers is their spherical-shaped structure. During synthesis, the size and shape of the dendrimer can be customized and controlled, so the finished macromolecule will have a specific "architecture" and terminal groups. These characteristics will determine its suitability for drug delivery, diagnostic imaging, and as a genetic material carrier. This review will focus initially on the unique properties of dendrimers and their use in biomedical applications, as antibacterial, antitumor, and diagnostic agents. Subsequently, emphasis will be given to their use in drug delivery for ocular diseases.
\end{abstract}

Keywords: nanoparticles, ocular diseases, encapsulation, macromolecule, diagnostic agent

\section{Introduction}

The efficient treatment of certain illnesses via optimization of drug delivery is a major goal of current scientific research. Recent advances in nanomedicine provide new tools for dealing with this challenge. Medical treatments based on nanostructured polymers have shown great potential as therapeutics in several central nervous disorders, such as Alzheimer's, Parkinson's, and epilepsy. Ocular diseases can also be treated more efficiently using nanostructured drug carriers. ${ }^{1}$ Polymers, either natural or synthetic, are materials upon which a specific area of nanomedicine is based on. The use of natural polymers, specifically the biodegradable ones, have aided considerably in the efficacy of drug delivery, as novel drug delivery systems. Nanobiopolymers, in particular, play a crucial role in this area by expanding the spectrum of pharmaceutical and biomedical applications. Dendrimers belong to a class of nanobiopolymers that are characterized as one of the most promising innovative polymeric nanocarriers for a variety of therapeutic procedures in target delivery systems. ${ }^{2}$

Dendrimers (from the Greek Dendron meaning tree), are discrete nanoparticles with "onion skin-like" branched layers. ${ }^{2,3}$ Dendrimers are defined as synthetic macromolecules characterized by a large number of branching points, a threedimensional globular shape, monodispersity, and nanometric size range. Their three-dimensional structure has a significant impact on their physical and chemical properties. In particular, their spherical shape and ability to penetrate cell walls easily, due to their size and lipophilicity, renders them ideal as drug delivery systems and are in general more preferable for these applications than the classic linear polymers. ${ }^{4}$ A macromolecular drug delivery moiety is used as the vehicle into or onto which a drug is attached to be carried to a specific location. Such unit vessels can be synthetic polymers (dendrimers, in this case) attached to antibodies, hormones, or liposomes. The loading and delivery of a specific drug in such a system depends on the properties
Correspondence: Nikos A Chaniotakis Laboratory of Analytical Chemistry, Department of Chemistry, University of Crete Voutes, 7I003 Heraklion,

Crete, Greece

$\mathrm{Tel}+302810545018$

Fax +302810545165

Email chaniotakis@uoc.gr
International Journal of Nanomedicine 2016:I I I-12

Dovepress

http://dx.doi.org/1 0.2147/IJN.S93069 (c) (i) (5) 2016 Kalomiraki et al. This work is published by Dove Medical Press Limited, and licensed under Creative Commons Attribution - Non Commercial (unported, v3.0) License. The full terms of the license are available at http://creativecommons.org/licenses/by-nc/3.0/. Non-commercial uses of the work are permitted without any further how to request permission may be found at: http://www.dovepress.com/permissions.php 
of the macromolecular carrier parameters, such as the number and type of active sites, loading space, external functional groups, lipophilicity, etc. In addition, an ideal drug carrier unit must be biochemically inactive and nontoxic. ${ }^{4,5}$ The drug must be protected and stabilized within the carrier until it is released to a specific location organ. Chemical modifications of the polymer properties allow for the optimization of such parameters in order to achieve the desired characteristics required for a specific application. In this review, we present the achievements of nanomedicine through dendrimers in the area of targeted drug delivery in medicine and pharmaceutics, with particular emphasis on the ocular system. ${ }^{2-5}$

\section{Structure of dendrimers}

Dendrimers have a characteristic architecture with a welldefined branched structure with a globular shape. Their shape and chemical composition allows for the existence of a large number of surface groups (Figure 1). These groups can be considered the active sites, which can be custom-tailored to provide a template for drug immobilization and grafting. A typical dendrimer consists of three major domains, as shown in Figure 1:

1. The core at the center of the dendrimer, which is the synthetic starting point and consists of an atom or more, usually a multifunctional molecule.

2. The branching units, which are covalently bound to the core molecule and are units whose repetition leads to a series of radially concentric layers called "generation".

3. The terminal functional groups, located at the surface of the dendritic architecture, and based on the synthetic route followed, as well as the generation of the dendrimer.

Since dendrimers grow and branch out by the attachment of monomeric units in a stepwise manner, a precise control on molecular size, shape, dimension, density, polarity, flexibility, and solubility is theoretically feasible. The choice of different branching units and surface functional end groups determine the final physical and chemical characteristics of the nanostructure. ${ }^{5}$ The early-generation

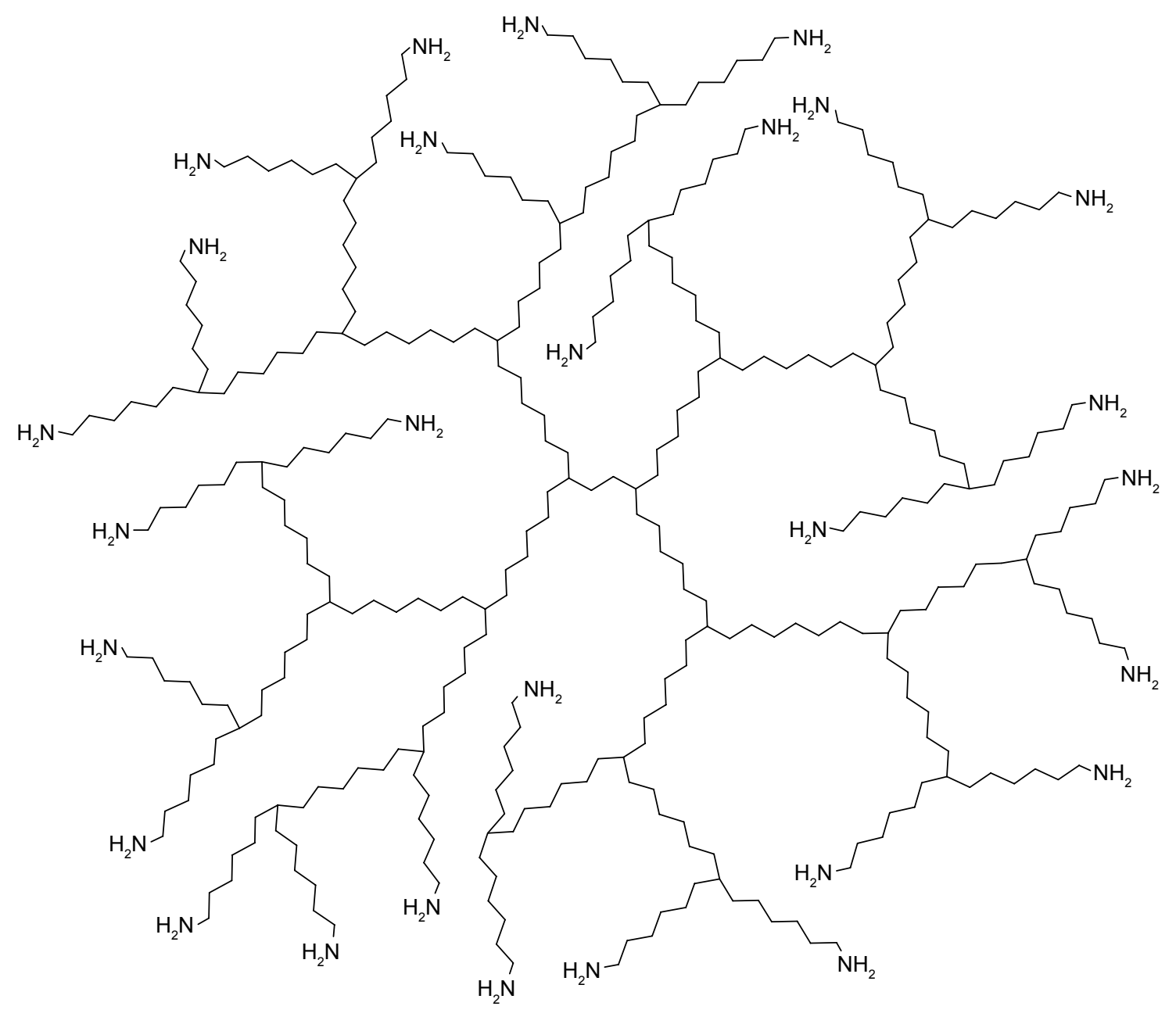

Figure I A schematic structure of dendrimers. 


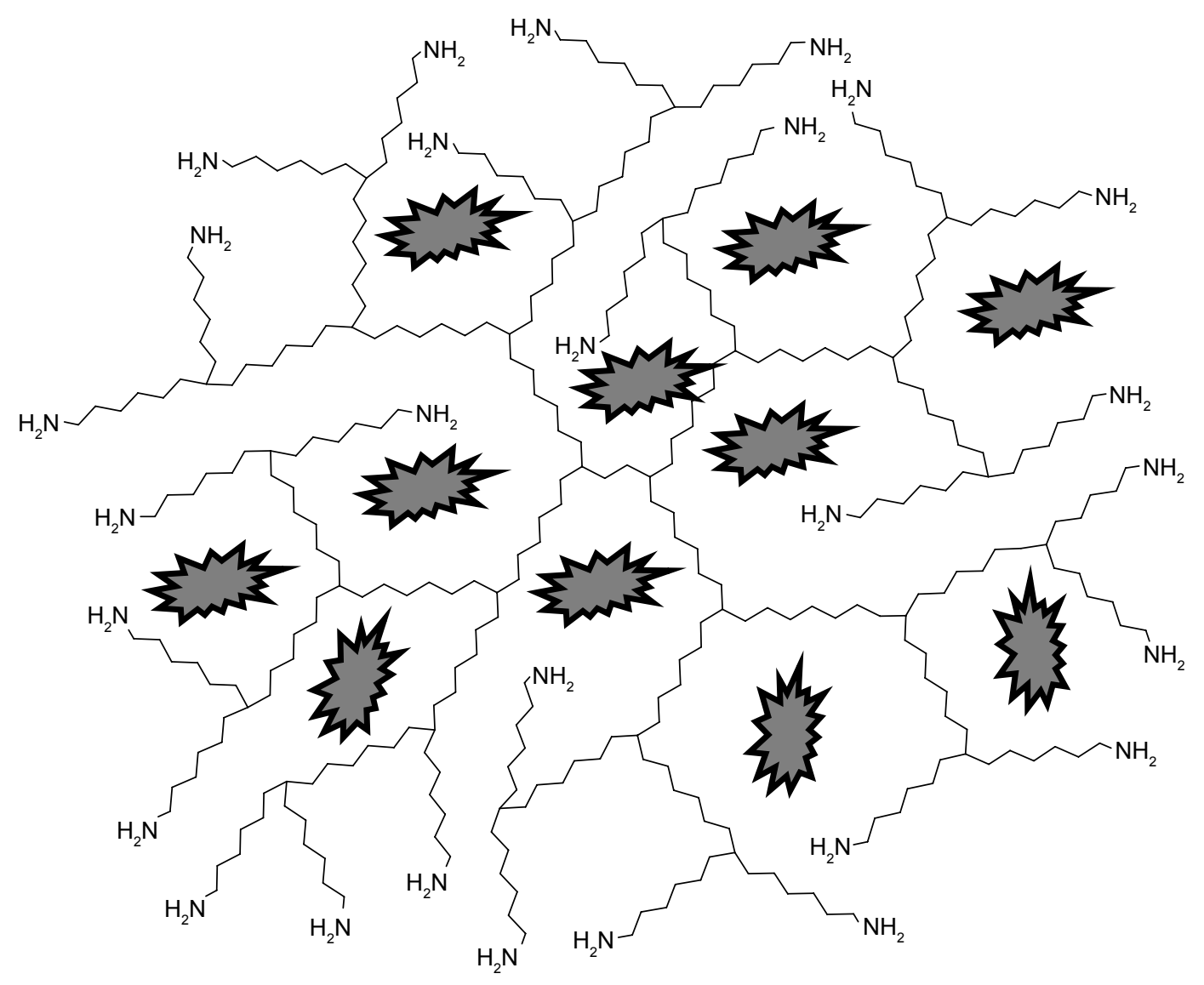

Figure 2 Physical encapsulation of the drug to dendrimer.

dendrimers have empty internal cavities and open configurations, which make it possible to encapsulate hydrophobic drugs (Figure 2). At this early synthetic stage, they have a high surface functional group density, and a high site-tosize ratio. The type and number of these functional groups provide dendrimers their characteristics for drug grafting and/or solubility. In addition, the types of outer surface functional groups are responsible for the observed reactivity and upon modification they can accommodate the guest molecule of interest.

\section{Physical and chemical properties of dendrimers}

The goal of nanomedicine is to control and handle biomacromolecular and supramolecular constructs that are critical to living cells in order to enhance the quality of human health. Dendrimers have a variety of unique properties that render them suitable for pharmaceutical applications. ${ }^{5-7}$ They are monodisperse macromolecular units and their synthetic procedure for specific size and molecular mass is well established. Their shape is well defined, and it is tightly packed. Dendrimers have well-controlled rheological properties due to their reproducible shape and size. Their intrinsic viscosity reaches a maximum at the fourth generation and then begins to decrease. The terminal groups can be controlled to have distinct characteristics, such as hydrophilic or lipophilic character, controlled solubility, miscibility, acidity, etc. Due to their spherical shape, dendrimers can encapsulate small size drugs, metals, or imaging moieties that can fit within their branches, and can interact via hydrogen bonding, lipophilicity, and charge interactions.

This encapsulation is a major advantage, as it reduces possible drug toxicity, increases its stability, and facilitates the targeted and controlled release. They are relatively biocompatible, especially the lower generation ones with anionic or neutral polar terminal surface groups. Their body excretion mode is a function of their nanoscale diameter, and can be well controlled and monitored. Different surface groups can be utilized to provide the means for modification and optimum biodistribution, receptor-mediated targeting, therapy dosage, or controlled release of drug. Finally, dendrimers provide a precisely controlled macromolecular surface, with a far lower cost base than proteins. 


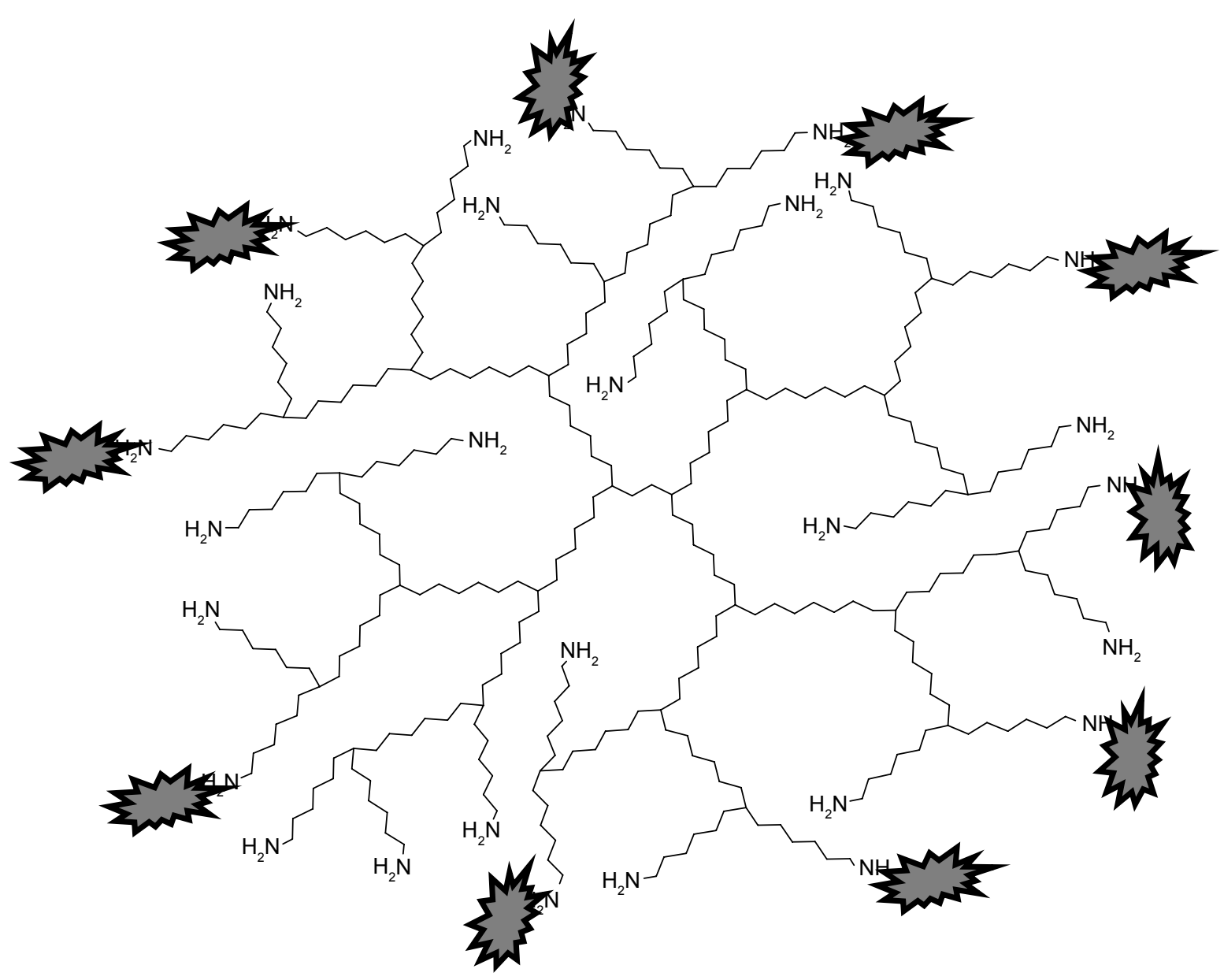

Figure 3 Conjugation of the drug to dendrimer.

\section{Interactions between drug molecules and dendrimers}

A dendrimer can interact in several ways with organic compounds, such as drugs. These interactions can be subdivided into two main categories: the physical and the chemical ones. They may involve simple encapsulation, hydrophobic interaction, or hydrogen bonding. The physical interaction is a nonbonding interaction between specific groups within dendrimer and the drug (Figure 2), and refers to the simple encapsulation of the molecule. ${ }^{8}$ This is due to the presence of the ellipsoidal or spheroidal shape, the empty internal cavities, and open nature of the architecture of dendrimers. The empty internal cavities have hydrophobic properties, which make possible its interaction with poorly soluble drugs through hydrophobic interactions. ${ }^{9,10}$

Novel polyester-co-polyether dendrimers were also synthesized having a hydrophilic core, which was composed of biocompatible moieties, such as butanetetracarboxylic and aspartic acid. The dendrons were synthesized by polyethylene glycol (PEG), dihydroxybenzoic or gallic acid, and PEG monomethacrylate. Dendrimers were formed by the coupling of these agents to the surface functional groups. The loading of rhodamine and $\beta$-carotene to polyester-co-polyether dendrimers can be achieved in a satisfactory manner and these dendrimers can be potent drug delivery systems. ${ }^{11}$

The chemical bonding involves two types of interactions between the drug and the dendrimer. The first type involves electrostatic interactions and the conjugation of the drug molecule to the dendrimer (Figure 3). The large number of ionizable functional groups (such as amine and carboxyl groups) and their high density on the surface of dendrimers can enhance the solubility of hydrophobic drugs. An ester bond formation between carboxylic acid dendrons and polyalcoholic cores ${ }^{12}$ can be performed by using uranium-based coupling agents, o-(Benzotriazol-1-yl)$\mathrm{N}, \mathrm{N}, \mathrm{N}^{\prime}, \mathrm{N}^{\prime}$-tetramethyluronium tetrafluoroborate (TBTU), ${ }^{13}$ o-(7-Azabenzotriazol-1-yl)-N,N, $\mathrm{N}^{\prime}, \mathrm{N}^{\prime}$ - tetramethyluronium tetrafluoroborate (TATU), ${ }^{14}$ and 1-([1-[Cyano-2-ethoxy-2oxoethylideneaminooxy]-dimethylamino-morpholino]) uranium hexafluorophosphate (COMU). ${ }^{15}$ Recent studies have shown that nonsteroidal anti-inflammatory drugs (NSAIDs) with carboxyl groups, including ibuprofen, ${ }^{16}$ piroxicam, ${ }^{17}$ and 
indomethacin, ${ }^{18}$ can form stable complexes with dendrimers through electrostatic interactions. ${ }^{19}$

The second type of interaction is based on the presence of active moieties that include PEG, $p$-amino benzoic acid, or biodegradable linkages, such as amide or ester bonds, through which the drugs can be covalently conjugated to dendrimers, forming a structure called "prodrug". Some drug molecules such as penicillin $\mathrm{V},{ }^{20}$ venlafaxine, ${ }^{21} 5$-aminosalicylic acid, ${ }^{22}$ naproxen, ${ }^{23}$ and propranolol ${ }^{24}$ have successfully conjugated with polyamidoamine (PAMAM) dendrimers. Furthermore, there are anticancer drugs such as cisplatin, ${ }^{25}$ doxorubicin (DOX), ${ }^{26}$ epirubicin, ${ }^{27}$ methotrexate, ${ }^{28}$ and paclitaxel ${ }^{29}$ that have successfully conjugated with PAMAM dendrimers. It is worth mentioning that the chemical bonding between drug molecule and dendrimer active sites allow for the precise drug release control, since the complex (drug-dendrimer) has a slower diffusion rate than that of the free drug. ${ }^{30}$

PEG is a polymer compound that is usually coupled to the surface of dendrimers resulting in the creation of a hydrophilic shell around the hydrophobic dendrimer. This PEGylation forms unimolecular micelles that can solubilize hydrophobic drugs in the interior of dendrimers and hydrophilic drugs in the PEG layer.

\section{Applications of dendrimers in nanomedicine}

The three unique architectural properties of a dendrimer, the core, the branching points, and the terminal functional groups, suggest drug dendrimers as ideal agents for a variety of therapeutic applications. In this review, the use of dendrimers in different biomedical applications will be discussed with emphasis given to their use in ocular diseases.

\section{Diagnostic applications}

The unique morphology of dendrimers makes them a promising candidate for diagnostic applications. They have been used efficiently as imaging agents in radiotherapy, $\mathrm{X}$-ray, and magnetic resonance imaging (MRI). ${ }^{31,32} \mathrm{In} \mathrm{X}$-ray technology, substances of high molecular weight are preferred over low molecular weight as the latter substances equilibrate readily within the extracellular fluid and intravascular compartments of the body. For this reason, they are used to detect disease-induced lesions or view organs such as kidneys, bones, tumors, and arteriosclerotic vasculature. Organometallic ( $\mathrm{Bi}$ and $\mathrm{Sn}$ ) complexes of dendrimers, dendrimers with PEG cores, and dendritic nanoparticles with heavy isotopes of iodine have been used for such applications. ${ }^{33-35}$

In the MRI technique, several complexes of gadolinium (Gd[III]) ions with high molecular weight compounds like albumin, dextran, polylysine, and dendrimers are explored. ${ }^{36-39}$ Nanoparticles are employed in order to generate tissue-specific contrast in tumors. Gadolinium-diethylene triamine penta-acetic acid (Gd-DTPA) polylysine contrast agents are effective in the diagnosis and staging of brain and other tumors. The contrast is generated not by targeting specific molecular aspects of the tumors, but by creating morphological changes to the tissue. ${ }^{37}$ A more recent study employed two contrast nanoparticles, namely targeted gadopentate-tetraazacyclo dodecane-tetra acetic acid-G5folic acid (Gd[III]-DOTA-G5-folic acid) and a not-targeted Gd(III)-DOTA-G5, to perform a pharmacokinetic study in human epidermoid carcinoma ([KB], xenograft tumors in mice), which overexpress the folate receptor. The results demonstrated that Gd(III)-DOTA-G5-folic acid dendrimer generated a statistically significant signal enhancement in tumors as compared with the signal obtained when the nontargeted Gd(III)-DOTA-G5 contrast agent was used. ${ }^{38}$

\section{Gene delivery applications}

Dendrimers with structural flexibility and hyperbranched architectures are suitable candidates for gene delivery operations due to the formation of more compact complexes with DNA. Flexible dendrimers and partially degraded high generation dendrimers are more preferable than high generation solid dendrimers in specific gene delivery procedures. Initially, the dendrimer-DNA complex is taken up in cells by endocytosis and consequently, an endosomal destabilization of electrostatically assembled dendrimerDNA complex takes place, which is followed by the subsequent release of DNA (Figure 4).$^{40}$ Studies have shown that polypropylene imine (PPI) generation 2 dendrimer binds efficiently to DNA, has low cytotoxicity to cells, and the in vitro gene transfer activity is optimized. ${ }^{41}$

The gallic acid-triethylene glycol dendrimers due to their high stability and agility are used as a gene delivery

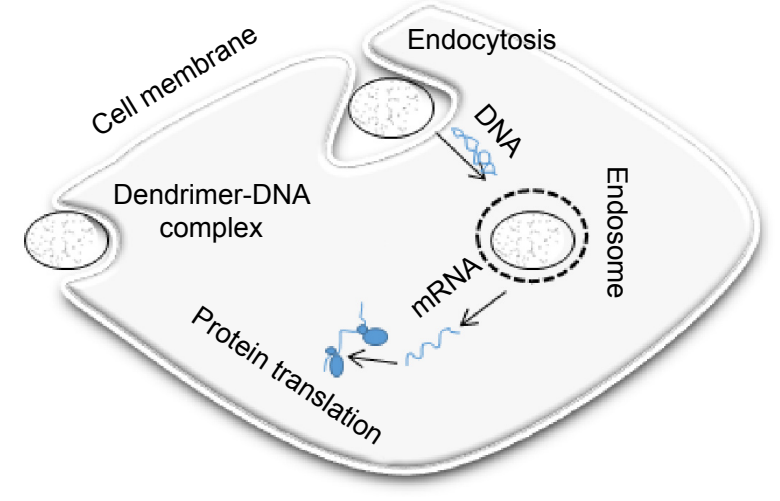

Figure 4 Dendrimer-mediated gene transfection.

Abbreviations: DNA, deoxyribonucleic acid; mRNA, messenger ribonucleic acid. 
system. Polyion complex micelles have a core-shell structure surrounded by a neutral hydrophilic corona, usually a PEG. These micelles are preferred to classic micelles because they are capable of delivering low molecular weight drugs, proteins, imaging agents, and nucleic acids. ${ }^{42}$

\section{Therapeutic applications}

\section{Antibacterial agents}

Dendrimers act as antibacterial agents due to their molecular surface functional group concentration. This property can be enhanced by functionalizing the surface groups with biologically active antimicrobial groups. Converting the dendrimer end groups to ammonium salts lead to the synthesis of dendrimer biocides. The electrostatic interaction between the positively charged dendrimer biocides and the negatively charged bacteria is responsible for the antibacterial actions of the dendrimers. Water-soluble dendrimers can host insoluble antibiotics (such as quinolones), improve their bioavailability, and thus their antibacterial properties. It was shown that the presence of PAMAM-based dendrimers increases the water solubility and the antimicrobial activities of nadifloxacin and prolifloxacin. ${ }^{43}$

There are many more paradigms employing dendrimers as antimicrobial agents and therapeutics. The creation of the SPL7013 Gel (VivaGel; Starpharma, Preston, Melbourne, Victoria, Australia) Platform is one such example. SPL7013 is the active ingredient of a polylysine dendrimer. SPL7013 is a topical antimicrobial agent that was shown to be effective against herpes simplex virus (HSV-1 and HSV-2) ${ }^{44}$ and human immunodeficiency virus (HIV) in vitro. ${ }^{45}$ Unformulated SPL7013 was also employed in animal model studies. When administered in mouse vaginas, it rendered a dose-related protection from HSV-2 infection. ${ }^{46}$ In macaque monkeys, SPL7013 gels administered in a single intravaginal application was found to be safe and protected macaques against vaginal simian HIV infection.

This microbicide was also examined in humans (Phase I clinical trial) and found to be well tolerated, after a daily intravaginal administration for 7 days, compared with placebo. ${ }^{47}$ Most importantly, it was shown that SPL7013 was not absorbed into the systemic circulation, reducing possible toxicity.

Polylysine dendrimers (second and fourth generation) designed specifically for HSV-1, HSV-2, and HIV were examined for their efficacy against the aforementioned viruses, with respect to their number of lysine branches. HIV-1 and HSV-2 structure-activity relationship studies of L-lysine dendrimers were performed in different strains of these viruses. The fourth-generation SPL7013 dendrimer afforded broad anti-HIV activity, provided at least 12-hour protection against HSV-2 in mouse retina and was not toxic. ${ }^{43}$
Efficacious topical microbicides, such as SPL7013 and VivaGel, are essential for the prevention of the transmission of HIV and HSV-2 and other genital infections in women. The data presented in the study by Tyssen et al, ${ }^{47}$ in addition to those of previous studies presented earlier in this review, support the need for further evaluation of VivaGel in clinical trials for the assessment of its therapeutic potential as an efficacious topical microbicide in women with genital infections, when administered intravaginally, either alone or in conjunction with other antiviral agents.

\section{Vaccines}

Dendrimers also act as carriers of antigens and immunostimulatory adjuvants that can be used to improve the efficiency of vaccine formulations. They can generate highly multimeric antigenic conjugates leading to a preparation that can be administered to human organisms without adverse or aggravating host responses, such as immune and inflammatory reactions. ${ }^{48,49}$

\section{Anticancer drugs}

The epidermal growth factor receptor is present in human gliomas and it is a potential target for the delivery of therapeutic agents to brain tumors. The chimeric monoclonal antibody cetuximab (IMC-C225) is considered as a boron delivery agent for neutron capture therapy. A heavily boronated fifth-generation PAMAM dendrimer (G5-B1100) linked to $\mathrm{C} 225$ and boron neutron capture therapy has been examined and it is being assessed for the treatment of intracerebral brain tumors. ${ }^{50}$

In addition, the covalent conjugation of DOX, a putative anticancer drug to a three-arm poly (ethylene oxide) dendrimer hybrid has also been examined. The study revealed a longer release time for the polymer-DOX conjugate compared with the free DOX. It has also been found that there was no important accumulation of the drug in vital organs (eg, liver, heart, and lungs) and that it is a highly water-soluble, nontoxic, and biocompatible polymer. The antitumor effect of DOX conjugated to a biodegradable polymer was verified in mice facing C-26 colon carcinomas. ${ }^{51}$

In an in vivo-targeted drug delivery study, the conjugation of folic acid to PEG-PAMAM dendrimers followed by the coupling of the anticancer drug methotrexate showed that a significant improvement in the therapeutic index of a targeted polymer-drug conjugate over the free drug (methotrexate) was achieved. ${ }^{52}$ This was shown to be possibly due to the decrease in toxicity and an increase in drug effectiveness. 
Intravenous injection of the dendrimer conjugates into immunodeficient mice with human KB tumors led to their longer survival. It was also shown that the drug conjugates internalized in the tumor cells 4 days after administration. In addition, it was also shown that this conjugate provided greater antitumor activity compared with non-PEGylated dendrimers.

The anticancer drug 5-fluorouracil has been encapsulated in PEGylated dendrimers. Studies revealed that the drug encapsulation increases according to the increase of the molecular weight of PEG and has lower cytotoxicity and hemolytic activity. ${ }^{53}$

\section{Analgesic and anti-inflammatory components of diseases}

As mentioned earlier, dendrimers are efficacious in improving drug solubility, control release, and drug-polymer conjugation (prodrugs). NSAIDs such as ketoprofen, ibuprofen, and diflunisal are used therapeutically to target the pain and inflammatory components of many diseases. The use of dendrimers to encapsulate different NSAIDs was shown to influence their pharmacokinetic properties, such as their solubility and absorption. Koc and Senel, ${ }^{54}$ employing polypolypropylene oxide core PAMAM dendrimers, showed that: 1) the core size; 2) different generations ( $\mathrm{G} 0-\mathrm{G} 5)$; and 3) concentration of PPO-PAMAM dendrimer influences the increase of drug solubility in aqueous solution of the NSAIDs. Under constant concentration and generation of the dendrimer, the solubility increased for the aforementioned NSAIDs in the following manner: ketoprofen $>$ diflunisal $>$ ibuprofen. The solubility-enhancement properties of PPO@ PAPAM dendrimers were shown to be four times higher than the ethylenediamine cored PAPAM dendrimers. These results recommend that the core size and properties of the dendrimer may play an important role in the increase of the solubility of the dendrimer.

\section{Oral drug delivery}

PAMAM-based dendrimers with or without surface modifications were studied for oral drug delivery. It was demonstrated that dendrimers with lauroyl groups could efficiently traverse epithelial monolayers via paracellular and transcellular pathways. ${ }^{55}$ Some examples of PAMAMbased dendrimers are: conjugates PAMAM with propranolol and prodrug of naproxen. PAMAM dendrimers were also assessed for pulmonary absorption of enoxaparin and were found to be promising vehicles for pulmonary delivery of therapeutics. ${ }^{56,57}$

\section{Dendrimers in ocular drug delivery}

Targeted drug delivery to either the anterior or the posterior segments of the eye for obtaining efficacious therapeutics for ocular diseases is still one of the major challenges of drug design. Current methods for drug delivery to the anterior part of the eye involve the topical route of administration, applied either as eye drop, suspension, or hydrogel formulations. These agents have low ocular bioavailability and efficacy due to early elimination, low permeability of the corneal epithelial membrane, and low corneal residence time. Drug administration to target diseases of the posterior segment of the eye, such as glaucoma, diabetic retinopathy (DR), age-related macular degeneration (ARMD), and genetically linked diseases such as retinitis pigmentosa is more challenging. The acellular nature of the vitreous and the longer diffusion distance toward the retina hampers the ability of drugs to reach their target and as such their bioavailability. ${ }^{58}$ Less than $5 \%$ of administered drug enters the eye and reach the retina. ${ }^{59}$ Ischemia-induced ocular diseases such as DR, ARMD, and glaucoma, are characterized by neovascular and/or neurodegenerative and neuroinflammatory components, which underlie the mechanisms leading to retinal cell death, suboptimal visual acuity, and blindness. Over the years, therapies for ischemic neovascular diseases have been focused on the regulation of the aberrant proliferation of blood vessels. Laser photocoagulation therapy is applied alone or in combination with verteporfin, a photosensitizing porphyrin (photodynamic therapy, ie, for the treatment of the wet form of ARMD). The technique of laser photocoagulation has limitations, namely it does not restore lost vision, it leads to an immediate reduction in central vision, and there is an approximate $50 \%$ chance that leakage will recur during the following 2 years. ${ }^{60}$ Photodynamic therapy, on the other hand, is effective in treating established pathological vessels, but it does not prevent new vessel formation. ${ }^{61}$

The proneovascular (angiogenic) agent vascular endothelium growth factor (VEGF) is considered to be the factor most responsible for the development of new vessels in diseases such as ARMD, choroidal neovascularization (CNV), and proliferative DR. Anti-VEGF drugs such as bevacizumab (Avastin, full-length recombinant humanized anti-VEGF monoclonal antibody; Genentech, Inc., South San Francisco, CA, USA), ranibizumab (Lucentis, an engineered, humanized, recombinant antibody fragment [Fab] active against all VEGF-A isoforms; Genentech/Novartis Ophthalmics, Basel, Switzerland), pegaptanib (Macugen, 28-nucleotide RNA aptamer that binds specifically to VEGF-A 165 isomer; Eyetech Inc., Cedar Knols, NJ, USA), and aflibercept (Eylea, 
VEGF Trap-Eye, recombinant fusion protein comprising the key VEGF-binding domains of human VEGF receptors 1 and 2; Regeneron, Tarrytown, NY, USA) are now being employed for the treatment of the aforementioned ocular diseases. ${ }^{62,63}$

The discovery of anti-VEGF therapy has given new hope to patients with wet ARMD, CNV, and proliferative DR, yet serious ocular adverse events are observed, the most common being conjunctival hemorrhage, eye pain, increased intraocular pressure, and intraocular inflammation. In addition, serious adverse events related to the injection procedure also occurred with an incidence rate of less than $1 \%$ and included endophthalmitis, retinal detachment, and traumatic cataracts. ${ }^{64}$

The development of new drug delivery systems of drugs that are either available or under investigation, with known pharmacodynamics for ocular diseases (antineovascular, anti-apoptotic/neuroprotective, or antineuroinflammatory) is of great importance and essential for the treatment of ocular diseases. The ideal ocular drug delivery system should be nonirritating, isotonic, biocompatible, and biodegradable. It should also prolong the release of the drug, reduce side effects, and target the desirable eye segment. Investigations of targeted drug-dendrimers for their use in different ocular diseases are discussed ahead.

\section{Dendrimers and glaucoma}

Glaucoma, an optic neuropathy, has recently been identified as a neurodegenerative disease with progressive ganglion cell death and visual loss. It is characterized by elevated intraocular pressure. The present available therapeutic treatments that target this ocular disease pertain to drugs that regulate the ocular hypertension, namely drugs that influence the synthesis or outflow of aqueous humor (cholinomimetics-muscarinic stimulants or cholinesterase inhibitors, $\alpha$-adrenergic receptor agonists, $\beta$-adrenergic receptor blockers, diuretics, and prostaglandins).

Drugs of the aforementioned categories are administered in drops, ointments, or gel, and orally (eg, acetazolamide). In order to increase the bioavailability of these agents and reduce their side effects, nanoparticles have been prepared for ocular delivery.

PAMAM dendrimers were examined for the putative use as ophthalmic vehicles for ocular delivery of pilocarpine nitrate (cholinomimetic). ${ }^{65}$ It was observed that the use of dendrimers with carboxylic or hydroxyl surface groups as drug carriers increased the bioavailability of pilocarpine nitrate in eyes of New Zealand albino rabbits and the time of drug residence. In the same study, it was shown that the antimuscarinic drug tropicamide combined with dendrimer solutions enhanced pupil dilatation (mydriasis), useful for ophthalmoscopic examination.

In a recent study, ${ }^{66}$ a photoreactive dendrimer (PAMAMPEG acrylate conjugate) hydrogel was synthesized and employed for the investigation of the pharmacokinetic properties of two antiglaucoma drugs, namely the hydrophobic $\alpha_{2}$-adrenergic agonist brimonidine and the hydrophilic $\beta$-adrenergic blocker timolol maleate, delivered into human corneal epithelial cells in vitro and bovine corneas ex vivo. This nanoparticle platform consists of hydrogel dendrimers carrying photoreactive groups, conjugated to terminal functional groups of the dendrimer. The exposure of this platform to a suitable wavelength of light activates the photoreactive groups resulting in their photocrosslinking and the formation of a hydrogel. The results obtained suggest that this dendrimer hydrogel is a substantial platform for the delivery of the two antiglaucoma drugs studied, since their solubility, sustained release, and uptake was significantly increased compared with phosphate-buffered saline-drug solution delivered as eye drops.

An even more advanced vehicle for topical delivery of antiglaucoma drugs is the nanoparticle platform of hybrid dendrimer hydrogel/poly (lactic-co-glycolic acid). The hybrid dendrimer nanoparticle platform (HDNP) platform consists of three domains: the PAMAM dendrimer core to encapsulate hydrophobic drugs, the Poly (Lactic-co-Glycolic Acid) (PLGA) nanoparticles to deliver either hydrophobic or hydrophilic drugs, and the PEG network to load hydrophilic drugs. The major advance of this novel nanoplatform is its ability to deliver simultaneously multiple drugs in the same dosages and release them in a slow manner with sustained efficacy. ${ }^{67}$ These platforms are very promising tools in ocular nanomedicine.

The encapsulation of another antiglaucoma drug, acetazolamide into PPI dendrimers (generation 5) was prepared and studies were performed in vivo to evaluate its ability to reduce the intraocular pressure when administered topically in male New Zealand albino rabbits. In addition, in vitro studies were performed to investigate the rate of release of acetazolamide. The results obtained recommend that the intraocular pressure was attenuated when the acetazolamide encapsulated into the PPI dendrimers was administered topically. In addition, it was shown that acetazolamide, in the formulation of dendrimer-drug, was released in a sustainable rate. Therefore, this study concluded that PPI dendrimers appear to be a promising ophthalmic carrier for the ocular delivery of acetazolamide in glaucoma treatment. ${ }^{59}$ 


\section{Dendrimers and neuroinflammatory ocular disorders}

As mentioned earlier, neuroinflammation is a key player in the pathophysiology of DR. It also plays a major role in the pathophysiology of photoreceptor and retinal pigment epithelium (RPE) cell loss in ARMD and retinitis pigmentosa. Iezzi et al ${ }^{68}$ employed hydroxyl-terminated PAMAM dendrimerdrug nanodevices to study the attenuation of neuroinflammation in two rat models (Royal College of Surgeons and Sprague Dawley) retinal degeneration. It was observed that the intravitreally administered PAMAM dendrimers had an intrinsic ability to be selectively localized within activated microglia. PAMAM dendrimers were shown to be localized in the outer retina of both retinal models of retinal degeneration using fluorescein isothiocyanate- and Cy5.5-labeled dendrimers and to remain in the target cells up to 35 days after administration. Fluocinolone acetonide, known to decrease photoreceptor cell death and retinal neuroinflammation, ${ }^{69,70}$ was conjugated to the PAMAM dendrimer and was shown to be released in a stable manner for more than 90 days. This conjugate provided significant neuroprotection to the photoreceptors and reduced neuroinflammation for 30 days. These findings are extremely important, since they provide hope for the development of new treatments to target the neurodegenerative and neuroinflammatory components of retinal disease.

\section{Dendrimers and age-related macular degeneration}

The growth factor VEGF is responsible for the development of new blood vessels (neovascularization or angiogenesis). Its levels increase under hypoxic or ischemic conditions. As mentioned earlier, anti-VEGF agents administered as therapeutics in ocular diseases characterized by neovascularization are very efficacious, but also have undesirable side effects. Antisense oligonucleotides have been investigated as therapeutics in various ocular diseases. ${ }^{71,72} \mathrm{~A}$ sense oligonucleotide (ODN-1) targeted to a VEGF sequence was shown to reduce VEGF levels produced in hypoxic RPE cells, in vitro. In addition, this ODN-1 injected intravitreally in an animal model of CNV led to the attenuation of $\mathrm{CNV}$, as assessed by fluorescein angiography. ${ }^{73} \mathrm{CNV}$ was induced by laser photocoagulation. ${ }^{74}$

The synthesis and use of lipophilic amino acid dendrimers (lipid-lysine) to deliver ODN-1 was accomplished. Positively charged dendrimers were used to deliver ODN-1 into the nucleus of cultured D407 RPE cells. All dendrimer-ODN-1 conjugates mediated the attenuation of VEGF expression during an initial 24-hour period (40\%-60\%). The transfection efficiencies of the different dendrimers produced were studied and the results indicated that the most effective structural combination was three branched chains of intermediate length with eight positive charges such as that found for dendrimer 4 . Therefore, taking this into account, in vivo studies were performed using dendrimer 4 and $7-\mathrm{ODN}-1$ conjugates. Fluorescein angiography demonstrated that both dendrimers attenuated in a statistically significant manner $(P<0.0001)$, the severity of laser-mediated $\mathrm{CNV}$ for up to 2 months postinjection. ${ }^{75}$

The distribution, uptake, and toxicity of dendrimer 4-ODN-1 conjugates delivered into the eye was examined over a period of 6 months, and the inhibition of laser-induced $\mathrm{CNV}$ also studied during this period (2, 4, and 6 months) was subsequently investigated in rats and rabbits. ${ }^{76}$ The major findings of this study suggest that the dendrimer-ODN-1 conjugate is efficacious for up to 4-6 months, and has no adverse effects. The investigators recommended ODN-1 as a putative therapeutic for treating neovascular/angiogenic eye diseases.

\section{Dendrimers and cornea wound healing}

Traumatic or surgical injury pertaining to ocular disorders may lead to cornea wounds. The cornea of the eye serves an important role in refracting and focusing light rays necessary for clear vision. It possesses unique characteristics, namely an orderly arrangement of stromal collagen fibrils and lack of blood vessels that result in transparency. Inadequate healing of the cornea after injury can result in decreased or loss of vision. The use of nylon sutures is presently used for wound repair. However, there are a number of complications that arise from suturing the cornea and research is presently aimed toward the design of different methods to repair corneal wounds, such as the use of adhesive materials. The use of dendrimers to develop corneal adhesives has been investigated. ${ }^{77}$

A variety of dendrimer adhesives consisting of generations 1, 2, and 3 (G1, G2, and G3) associated with PEG, glycerol, and succinic acid were developed in order to repair the corneal wounds. Ocular adhesives were formed by a photocrosslinking reaction or by a peptide ligation reaction to couple the individual dendrimers together. The specific polymer used was a dendritic linear copolymer composed of PEG, glycerol, and succinic acid. The polymer was modified to contain terminal methacrylate groups and dissolved in a neutral aqueous solution. A cross-linked network was formed upon exposure of ([G1]-PGLSA-methacrylate)2-PEG 
to visible light. Linear incisions (4.1 mm thickness) were obtained, with the use of a keratome knife, in the central cornea of enucleated human eyes, ex vivo. The hydrogel that sealed the wound was obtained by the application of the photocrosslinkable adhesive to the laceration followed by irradiation with visible light. ${ }^{78-80}$

In a recent study, the properties of hydrogels prepared from the reaction of a peptide dendron (Lys3Cys4) with three different types of homobifunctional aldehyde-terminated PEG macromers: PEG-propionaldehyde (P-Ald PEG), PEGbutyraldehyde (B-Ald PEG), and PEG-2-oxoethyl succinate (E-Ald PEG) were studied in order to investigate the properties of pseudoproline or thiazolidine linkages. ${ }^{81}$

The adhesive strength of P-Ald hydrogels was weaker than the B-Ald and E-Ald hydrogels and its degradation was shown to be within hours of cross-linking, while the degradation times of B-Ald and E-Ald hydrogels were 1.5 and 24 weeks, respectively. It was also shown that the cysteineterminated Lys3Cys4 dendron reacted with the butyraldehyde poly ethylene glycol (BPEG) upon mixing to form a thiazolidine linkage, which is susceptible to hydrolysis. ${ }^{82}$

The E-Ald hydrogels were cross-linked by the formation of a thiazolidine linkage, which spontaneously rearranges to form the more stable pseudoproline linkages. As a result, these hydrogels are relatively slow to degrade due to the stability of pseudoproline cross-links with its amide bond in comparison to the reversible thiazolidine linkage. ${ }^{83}$ Regarding toxicity, B-Ald and E-Ald hydrogels showed no cytotoxicity to rabbit corneal fibroblasts. At high polymer concentrations, they did show moderate toxicity to NIH3 T3 fibroblasts. The constituent macromers were not found to be cytotoxic to rabbit corneal fibroblasts and NIH-3T3 fibroblasts at low concentrations.

The results of this study demonstrated the feasibility of the three hydrogel adhesives for cornea wound repair. They appeared to be useful in closing full-thickness ex vivo $4.1 \mathrm{~mm}$ central corneal lacerations well enough to withstand intraocular pressures as high as or higher than those of wounds closed with a single suture. Different properties of these hydrogels, such as mechanical properties and degradation times, may be able to be designed to suit a variety of wounds, ranging from quick-healing corneal incisions to slow-healing corneal transplants. Future research in this area may lead to the development of application-specific hydrogel adhesives for the repair of a variety of ocular wounds.

\section{Conclusion}

In this review, different paradigms were presented pertaining to the development of different dendrimers to generate diagnostic applications (eg, MRI tissue-specific contrast) or dendrimer-DNA complexes for gene delivery applications. In addition, specific studies were presented pertaining to dendrimer delivery of therapeutic agents having antibacterial and anticancer effects, as well as NSAIDs. Emphasis was given to the use of dendrimer formulations as therapeutics in ocular diseases with the hope that nanocarriers of existing drugs for the treatment of neovascularization (anti-VEGFs and ARMD) will improve target delivery and afford a better pharmacokinetic profile. In addition, the recent discovery of the role of multiple components in the pathophysiology of retinal diseases such as glaucoma and DR (eg, neurodegeneration and neuroinflammatory components), which are not treatable even today, make the development of new therapeutic targets and new delivery systems essential.

The therapeutics for all diseases either mentioned or not in this review will benefit from dendrimer formulations for drug delivery because of 1) improvement of the pharmacokinetic properties of drugs; 2) increase in their efficacy; and $3)$ reduction of their side effects.

\section{Disclosure}

The authors report no conflicts of interest in this work.

\section{References}

1. Xu L, Zhang H, Wu Y. Dendrimer advances for the central nervous system delivery of therapeutics. ACS Chem Neurosci. 2014;5(1):2-13.

2. Kesharwani P, Jain K, Jain NK. Dendrimer as nanocarriers for drug delivery. Prog Polym Sci. 2014;39:268-307.

3. Kannan RM, Nance E, Kannan S, Tomalia DA. Emerging concepts in dendrimer-based nanomedicine: from design principles to clinical applications. J Intern Med. 2014;276(6):579-617.

4. Tolia GT, Choi HH. The role of dendrimers in topical drug delivery. Pharm Technol. 2008;32(11):88-98.

5. Semwal R, Semwal DK, Madan AK, Paul P, Mujaffer F, Badoni R. Dendrimers: a novel approach for drug targeting. J Pharm Res. 2010; 3(9):2238-2247.

6. Tomalia DA, Reyna LA, Svenson S. Dendrimers as multi-purpose nanodevices for oncology drug delivery and diagnostic imaging. Biochem Soc Trans. 2007;35:61-67.

7. Tomalia DA. Birth of a new macromolecular architecture: dendrimers as quantized building blocks for nanoscale synthetic polymer chemistry. Prog Polym Sci. 2005;30:294-324.

8. Madaan K, Kumar S, Poonia N, Lather V, Pandita D. Dendrimers in drug delivery and targeting: drug-dendrimer interactions and toxicity issues. J Pharm Bioallied Sci. 2014;6(3):139-150.

9. Pillai O, Panchagnula R. Polymers in drug delivery. Curr Opin Chem Biol. 2001;5:447-451.

10. D'Emanuele A, Attwood D. Dendrimer-drug interactions. Adv Drug Deliv Rev. 2005;57:2147-2162.

11. Jean-d'Amour Twibarine T, Grindley B. Polyester dendrimers: smart carriers for drug delivery. Polymers. 2014;6:179-213.

12. Twibarine JK, Grindley TB. Efficient and controllably selective preparation of esters using uranium-based coupling agents. Org Lett. 2011; 13:2988-2991.

13. Knorr R, Trzeciak A, Bannwarth W, Gillessen D. New coupling reagents in peptide chemistry. Tetrahedron Lett. 1989;30:1927-1930.

14. Carpino LA. 1-Hydroxy-7-azabenzotriazole. An efficient peptide coupling additive. J Am Chem Soc. 1993;115:4397-4398. 
15. El-Faham A, Subiros Funosas R, Prohens R, Albericio F. COMU: a safer and more effective replacement for benzotriazole-based uranium coupling reagents. Chem Eur J. 2009;15:9404-9416.

16. Kolhe P, Misra E, Kannan RM, Kannan S, Lieh-Lai M. Drug complexation, in vitro release and cellular entry of dendrimers and hyperbranched polymers. Int J Pharm. 2003;259:143-160.

17. Prajapati RN, Tekade RK, Gupta U, Gajbhiye V, Jain NK. Dendrimermediated solubilization, formulation development and in vitro-in vivo assessment of piroxicam. Mol Pharm. 2009;6:940-950.

18. Chauhan AS, Sridevi S, Chalasani KB, et al. Dendrimer-mediated transdermal delivery: enhanced bioavailability of indomethacin. J Control Release. 2003;90:335-343.

19. Beezer AE, King AS, Martin IK, Mitchel JC, Twyman LJ, Wain CF. Dendrimers as potential drug carriers; encapsulation of acidic hydrophobes within water soluble PAMAM derivatives. Tetrahedron. 2003;59:3873-3880

20. Yang H, Lopina ST. Penicillin V-conjugated PEG-PAMAM star polymers. J Biomater Sci Polym Ed. 2003;14:1043-1056.

21. Yang H, Lopina ST. Extended release of a novel antidepressant, venlafaxine, based on anionic polyamidoamine dendrimers and poly (ethylene glycol)-containing semi-interpenetrating networks. J Biomed Mater Res A. 2005;72:107-114.

22. Wiwattanapatapee R, Lomlim L, Saramunee K. Dendrimers conjugates for colonic delivery of 5-aminosalicylic acid. J Control Release. 2003;88:1-9.

23. Najlah M, Freeman S, Attwood D, D'Emanuele A. In vitro evaluation of dendrimer prodrugs for oral drug delivery. Int J Pharm. 2007;336: 183-190.

24. D'Emanuele A, Jevprasesphant R, Penny J, Attwood D. The use of a dendrimer-propranolol prodrug to bypass efflux transporters and enhance oral bioavailability. J Control Release. 2004;95:447-453.

25. Malik N, Evagorou EG, Duncan R. Dendrimer-platinate: a novel approach to cancer chemotherapy. Anticancer Drugs. 1999;10:767-776.

26. Kaminskas LM, McLeod VM, Kelly BD, et al. A comparison of changes to doxorubicin pharmacokinetics, antitumor activity, and toxicity mediated by PEGylated dendrimer and PEGylated liposome drug delivery systems. Nanomedicine. 2012;8:103-111.

27. Pasut G, Scaramuzza S, Schiavon O, Mendichi R, Veronese FM. PEGepirubicin conjugates with high drug loading. J Bioact Compat Polym. 2005;20:213-230.

28. Soto-Castro D, Cruz-Morales JA, Ramírez Apan MT, Guadarrama P. Solubilization and anticancer-activity enhancement of methotrexate by novel dendrimeric nanodevices synthesized in one-step reaction. Bioorg Chem. 2012;41-42:13-21.

29. Zhou Z, D'Emanuele A, Attwood D. Solubility enhancement of paclitaxel using a linear-dendritic block copolymer. Int J Pharm. 2013; 452:173-179.

30. Aulenta F, Hayes W, Rannard S. Dendrimers: a new class of nanoscopic containers and delivery devices. Eur Polym J. 2003;39: 1741-1771.

31. Oliveira JM, Salgado AJ, Sousa N, Mano JF, Reis RL. Dendrimers and derivatives as a potential therapeutic tool in regenerative medicine strategies - a review. Prog Polym Sci. 2010;35:1163-1194.

32. Jang WD, Selim KMK, Lee CH, Kang IK. Bioinspired application of dendrimers: from bio-mimicry to biomedical applications. Prog Polym Sci. 2009;34:1-23.

33. Wu C, Brechbiel MW, Kozak RW, Gansow OA. Metal-chelatedendrimer-antibody constructs for use in radioimmunotherapy and imaging. Bioorg Med Chem Lett. 1994;4:449-454.

34. Krause W, Hackmann-Schlichter N, Maier FK, Muller R. Dendrimers in diagnostics. Top Curr Chem. 2000;210:261-308.

35. Yordanov AT, Lodder AL, Woller EK, et al. Novel iodinated dendritic nanoparticles for computed tomography (CT) imaging. Nano Lett. 2002; 2:595-599.

36. Schuhmann-Giampieri G, Schmitt-Willich H, Frenzel T, Press WR, Weinmann HJ. In vivo and in vitro evaluation of gadolinium-DTPApolylysine as a macromolecular contrast agent for magnetic resonance imaging. Invest Radiol. 1991;26:969-974.
37. Swanson SD, Kukowska-Latallo JF, Patri AK, et al. Targeted gadolinium-loaded dendrimer nanoparticles for tumor-specific magnetic resonance contrast enhancement. Int J Nanomedicine. 2008;3(2): 201-210.

38. Swanson SD, Rosen MS, Agranoff BW, Coulter KP, Welsh RC, Chupp TE. Brain MRI with laser-polarized 129Xe. Magn Reson Med. 1997;38:695-698.

39. Swanson SD, Rosen MS, Coulter KP, Welsh RC, Chupp TE. Distribution and dynamics of laser-polarised (129)Xe magnetization in vivo. Magn Reson Med. 1999;42:1137-1145.

40. Svenson S, Tomalia DA. Dendrimers in biomedical applications reflections on the field. Adv Drug Deliv Rev. 2005;57:2106-2129.

41. Zimselmeyer BH, Mackay SP, Schatzlein AG, Uchegbu IF. The lowergeneration polypropylenimine dendrimers are effective gene-transfer agents. Pharm Res. 2002;19(7):960-967.

42. Sousa-Herves A, Novoa-Carballal R, Riguera R, Fernandez-Megia E. GATG dendrimers and PEGylated block copolymers: from synthesis to bioapplications. AAOS J. 2014;16(5):948-961.

43. Cheng Y, Qu H, Ma M, et al. Polyamidoamine (PAMAM) dendrimers as biocompatible carriers of quinolone antimicrobials: an in vitro study. Eur J Med Chem. 2007;42:1032-1038.

44. Gong E, Matthews B, McCarthy T, et al. Evaluation of dendrimer SPL7013, a lead microbicide candidate against herpes simplex viruses. Antiviral Res. 2005;68:139-146.

45. Dezzutti CS, James VN, Ramos A, et al. In vitro comparison of topical microbicides for prevention of human immunodeficiency virus type 1 transmission. Antimicrob Agents Chemother. 2004;48:3834-3844.

46. Bernstein DI, Stanberry LR, Sacks S, et al. Evaluations of unformulated and formulated dendrimer-based microbicide candidates in mouse and guinea pig models of genital herpes. Antimicrob Agents Chemother. 2003;47:3784-3788.

47. Tyssen D, Henderson SA, Johnson A, et al. Structure activity relationship of dendrimer microbicides with dual action antiviral activity. PLoS One. 2010;5(8):e12309.

48. Kaufmann SHE. The contribution of immunology to the rational design of novel antibacterial vaccines. Nat Rev Microbiol. 2007;5(7): 491-504.

49. Heegaard PM, Boas U, Sorensen NS. Dendrimers for vaccine and immunostimulatory uses. A review. Bioconjug Chem. 2010;21(3): 405-418.

50. Wu G, Barth RF, Yang W, et al. Site-specific conjugation of boroncontaining dendrimers to anti-EGF receptor monoclonal antibody cetuximab (IMC-C225) and its evaluation as a potential delivery agent for neutron capture therapy. Bioconjug Chem. 2004;15(1):185-194.

51. Medina SH, El-Sayed ME. Dendrimers as carriers for delivery of chemotherapeutic agents. Chem Rev. 2009;109(7):3141-3157.

52. Kukowska-Latallo JF, Candido KA, Cao Z, et al. Nanoparticle targeting of anticancer drug improves therapeutic response in animal model of human epithelial cancer. Cancer Res. 2005;65(12):5317-5324.

53. Bhadra D, Bhadra S, Jain S, Jain NK. A PEGylated dendritic nanoparticulate carrier of fluorouracil. Int J Pharm. 2003;257:111-124.

54. Koc FE, Senel M. Solubility enhancement of Non-Steroidal AntiInflammatory Drugs (NSAIDS) using polypolypropylene oxide core PAMAM dendrimers. Int J Pharm. 2013;451:18-22.

55. Duan X, McLaughlin C, Griffith M, Sheardown H. Biofunctionalization of collagen for improved biological response: scaffolds for corneal tissue engineering. Biomaterials. 2007;28(1):78-88.

56. Grinstaff MW. Designing hydrogel adhesives for corneal wound repair. Biomaterials. 2007;28(35):5205-5214.

57. Kang SJ, Durairaj C, Kompella UB, O’Brien JM, Grossniklaus HE. Subconjunctival nanoparticle carboplatin in the treatment of murine retinoblastoma. Arch Ophthalmol. 2009;127(8):1043-1047.

58. Yavuz B, Bozdağ Pehlivan S, Ünlü N. Dendrimeric systems and their applications in ocular drug delivery. Sci World J. 2013; Article ID 732340:1-13.

59. Mishra V, Jain NK. Acatazolamide encapsulated dendritic nano-architectures for effective glaucoma management in rabbits. Int J Pharm. 2014;461:380-390. 
60. Fine SL, Berger JW, Maguire MG, Ho C. Age-related macular degeneration. New Engl J Med. 2000;342:483-492.

61. van Wijngaarden P, Coster DJ, Williams KA. Inhibitors of ocular neovascularization: promises and potential problems. JAMA. 2005;293(12):1509-1513. doi:10.1001/jama.293.12.1509.

62. Osaadon P, Fagan XJ, Lifshitz T, Levy J. A review of anti-VEGF agents for proliferative diabetic retinopathy. Eye (Lond). 2014;28:510-520. doi:10.1038/eye.2014.13.

63. Peden MC, Suner IJ, Hammer ME, Grizzard WS. Long-term outcomes in eye receiving fixed-interval dosing of anti-vascular endothelial growth factor agents for wet age-related macular degeneration. $\mathrm{Oph}-$ thalmology. 2015;122:803-808.

64. Vasilaki A, Thermos K. Somatostatin analogues as therapeutics in retinal disease. Pharmacol Ther. 2009;122(3):324-333.

65. Vandamme TL, Brobeck L. Poly(amidoamine) dendrimers as ophthalmic vehicles for ocular delivery of pilocarpine nitrate and tropicamide. J Control Release. 2005;102:23-38.

66. Holden CA, Tyagi P, Thakur A, et al. Polyamidoamine dendrimer hydrogel for enhanced delivery of antiglaucoma drugs. Nanomedicine. 2012;8(5):776-783.

67. Yang H, Leffler CT. Hybrid dendrimer hydrogel/poly(lactic-co-glycolic acid) nanoparticle platform: an advanced vehicle for topical delivery of antiglaucoma drugs and a likely solution to improving compliance and adherence in glaucoma management. $J$ Ocul Pharmacol Ther. 2013;29(2):166-172.

68. Iezzi R, Guru BR, Glybina IV, Mishra MK, Kennedy A, Kannan RM. Dendrimer-based targeted intravitreal therapy for sustained attenuation of neuroinflammation in retinal degeneration. Biomaterials. 2012;33(3):979-988.

69. Glybina IV, Kennedy A, Ashton P, Abrams GW, Iezzi R. Intravitreous delivery of the corticosteroid fluocinolone acetonide attenuates retinal degeneration in S334ter-4 rats. Invest Ophthalmol Vis Sci. 2010;51(8):4243-4252.

70. Glybina IV, Kennedy A, Ashton P, Abrams GW, Iezzi R. Photoreceptor neuroprotection in RCS rats via low-dose intravitreal sustaineddelivery of fluocinolone acetonide. Invest Ophthalmol Vis Sci. 2009; 50(10):4847-4857.

71. Alama A, Barbieri F, Cagnoli M, Schettini G. Antisense oligonucleotides as therapeutic agents. Pharmacol Res. 1997;36(3):171-178.
72. Boyer MI, Watson JT, Lou J, Manske PR, Gelberman RH, Cai SR. Quantitative variation in vascular endothelial growth factor mRNA expression during early flexor tendon healing: an investigation in a canine model. J Orthop Res. 2001;19(5):869-872.

73. Shen WY, Garrett KL, Wang CG, et al. Preclinical evaluation of a phosphorothioate oligonucleotide in the retina of rhesus monkey. Lab Invest. 2002;82(2):167-182.

74. Grossniklaus HE, Kang SJ, Berglin L. Animal models of choroidal and retinal neovascularization. Prog Retin Eye Res. 2010;29:500-519.

75. Marano RJ, Wimmer N, Kearns PS, et al. Inhibition of in vitro VEGF expression and choroidal neovascularization by synthetic dendrimer peptide mediated delivery of a sense oligonucleotide. Exp Eye Res. 2004;79(4):525-535.

76. Marano RJ, Toth I, Wimmer N, Brankov M, Rakoczy PE. Dendrimer delivery of an anti-VEGF oligonucleotide into the eye: a long-term study into inhibition of laser-induced CNV, distribution, uptake and toxicity. Gene Ther. 2005;12(21):1544-1550.

77. Grinstaff MW. Designing hydrogel adhesives for corneal wound repair. Biomaterials. 2007;28(35):5205-5214.

78. Carnahan MA, Middleton C, Kim J, Kim T, Grinstaff MW. Hybrid dendritic-linear polyester-ethers for in situ photopolymerization. $J \mathrm{Am}$ Chem Soc. 2002;124:5291-5293.

79. Grinstaff MW. Biodendrimers: new polymeric materials for tissue engineering. Chem Eur J. 2002;8:2838-2846.

80. Velazquez AJ, Carnahan MA, Kristinsson J, Stinnett S, Grinstaff MW, Kim T. New dendritic adhesives for sutureless ophthalmic surgeries: in vitro studies of corneal laceration repair. Arch Ophthalmol. 2004; 122:867-870.

81. Oelker AM, Berlin JA, Wathier M, Grinstaff MW. Synthesis and characterization of dendron cross-linked PEG hydrogels as corneal adhesives. Biomacromolecules. 2011;12(5):1658-1665.

82. Fife TH, Natarajan R, Shen CC, Bembi R. Mechanism of thiazolidine hydrolysis. Ring opening and hydrolysis of 1,3-thiazolidine derivatives of p-(dimethylamino)cinnamaldehyde. J Am Chem Soc. 1991; 113(8):3071-3079.

83. Liu CF, Tam JP. Peptide segment ligation strategy without use of protecting groups. Proc Natl Acad Sci U S A. 1994;91:6584-6588.
International Journal of Nanomedicine

\section{Publish your work in this journal}

The International Journal of Nanomedicine is an international, peerreviewed journal focusing on the application of nanotechnology in diagnostics, therapeutics, and drug delivery systems throughout the biomedical field. This journal is indexed on PubMed Central, MedLine, CAS, SciSearch ${ }^{\circledR}$, Current Contents ${ }^{\circledR} /$ Clinical Medicine,

\section{Dovepress}

Journal Citation Reports/Science Edition, EMBase, Scopus and the Elsevier Bibliographic databases. The manuscript management system is completely online and includes a very quick and fair peer-review system, which is all easy to use. Visit http://www.dovepress.com/ testimonials.php to read real quotes from published authors. 